\title{
The Differentiation of an Indefinite Integral.
}

\author{
By J. M. WhitTaker.
}

(Received 15th May 1925. Read 5th June 1925.)

1. Theorem 1 needs very little explanation. It is the converse of the well known theorem * that the indefinite integral $F(x)$ of a function $f(x)$ possesses a derivate on the right at every point at which $f(x+0)$ exists. If $f(x+0)$ does not exist, nothing can be said as to the existence or otherwise of $F_{+}(x)$; but in a general way we might expect that the integral of a function which oscillates comparatively slowly, say $\sin (\log x)$ at $x=0$, would be more likely to possess a derivate than that of a function which oscillates more rapidly, say $\sin \frac{1}{x}$. It appears from Theorem 1 that this is not by any means the case. In fact the integral of $\sin (\log x)$ has not a definite derivate at $x=0$ while that of $\sin \frac{1}{x}$ bas such a derivate. $\dagger$

The theorem is very similar in character to one due to $\mathbf{M r}$ Hardy concerning summable series.I The relation between $f(x+0)$ und $F_{+}(x)$ is indeed just the same as that between the sum and the sum $(C 1)$ of an infinite series.

2. Throrkm 1. Let $f(x)$ be continuous at all points of the interval $(0, a)$ except possibly the point $x=0$, and let the integral of $f(x)$ from $\epsilon$ to $x,(0<\epsilon<x \leq a)$, tend to a limit $F(x)$ as $\epsilon$ tonds to zero.

- Honson. Functions of a Real Variable. 2nd Edition, I. p. 454.

+ Cf. Lazshui Narayan, Bull. Calcutta Math. Soc., 8 (1916-17) p. 71.

$\ddagger$ Cf. Whittakrer and Watson. Modern Analysis, 3rd Edition, p. 156. 


\section{7}

Then $f(+0)$ exists and $=F_{+}(0)$ if

(i) $F_{+}(0)$ exists.

(ii) $(\alpha) \underline{D_{+}} f(x)<\frac{K}{x}$

$\left(0<x \leq x_{0}\right)$

or $\quad(\beta) \bar{D}_{+} f(x)>-\frac{K}{x}$

$\log x \sin \frac{1}{x}$ is an example of an unbounded function which satisfies all the conditions except (ii).

Writing

$$
g(x)=f(x)-F_{+}(0)
$$

we have to prove that each of the hypotheses (ii), say ( $\alpha$ ), combined with

$$
\int_{0}^{x} g(t) d t=x o(1) \quad \text { as } x \rightarrow 0
$$

implies

$$
g(x)=o(1)
$$

If this is not the case there must be a number $h$ such that in overy interval $0 \leq x \leq x_{1}$ there is a point $\xi$ at which either

(a) $g(\xi)>h$, or $(b) g(\xi)<-h$.

Take (a), and chose $K$ so large that

$$
\frac{h}{K}<1 \text {. }
$$

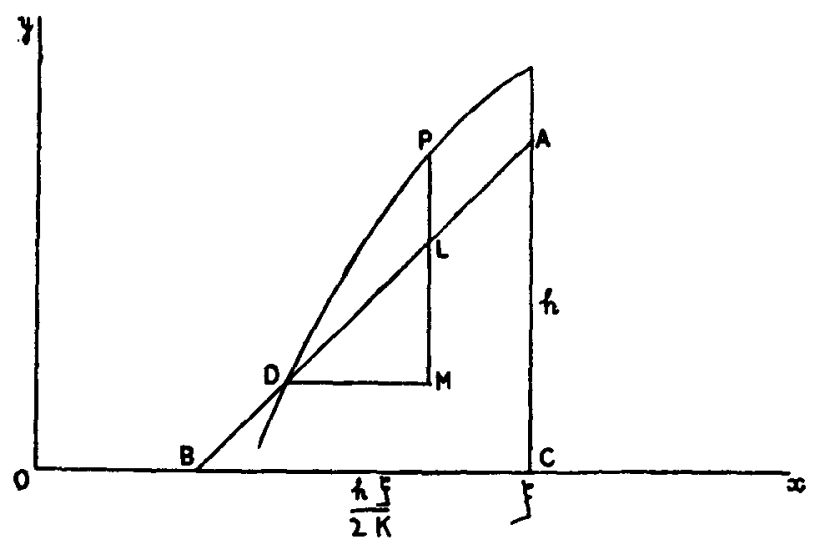

Fig. 1. 
We consider only points $x_{1}$ to the left of $x_{0}$.

From the point $A(\xi, h)$ draw a line $A B$ whose slope is $\frac{2 K}{\xi}$. Then the curve $y=g(x)$ must lie above this line. For if not let $\eta$ be the abscissa of $D$, the nearest point to $A$ at which the curve cuts the line. The existence of a nearest point follows from the sontinuity of $g(x)$. Then if $P$ be on the curve between $D$ and $A$ it is clear from Fig. 1 that

$$
\text { or } \quad \frac{g(x)-g(\eta)}{x-\eta}>\frac{2 K}{\xi} \quad(\eta<x<\xi)
$$

and taking bounds

$$
\underline{D}_{+} f(\eta) \geq \frac{2 K}{\xi}>\frac{K}{\eta}
$$

since

$$
\eta \geq \xi-\frac{h \xi}{2 K}>\xi-\frac{1}{2} \xi=\frac{1}{2} \xi
$$

This contradicts $(\alpha)$. Since therefore the curve must lie wholly above the line $A B$ it is clear that

$$
\int_{\xi_{1}}^{\xi} g(t) d t>\triangle A B C^{\gamma}=\frac{h^{2}}{4 \bar{K}} \xi
$$

But by $(2 \cdot 1)$

$$
\begin{aligned}
\left|\int_{\xi_{1}}^{\xi} g(t) d t\right| & \leq\left|\int_{0}^{\xi} g(t) d t\right|+\left|\int_{0}^{\xi_{1}} g(t) d t\right| \\
& =\xi o(1)+\xi_{1} \circ(1) \\
& =\xi \circ(1) .
\end{aligned}
$$

Thus for a set of values of $\xi$ tending to zero

$$
\xi \circ(1)>\frac{h^{2}}{4 K} \xi
$$

The contradiction implies that hypothesis $(a)$ is untenable. 
Consider now the consequences of $(b)$. There must exist a point $\bar{x}$ such that if $0<x_{1} \leq \bar{x}$ there is at least one point $\xi$ between

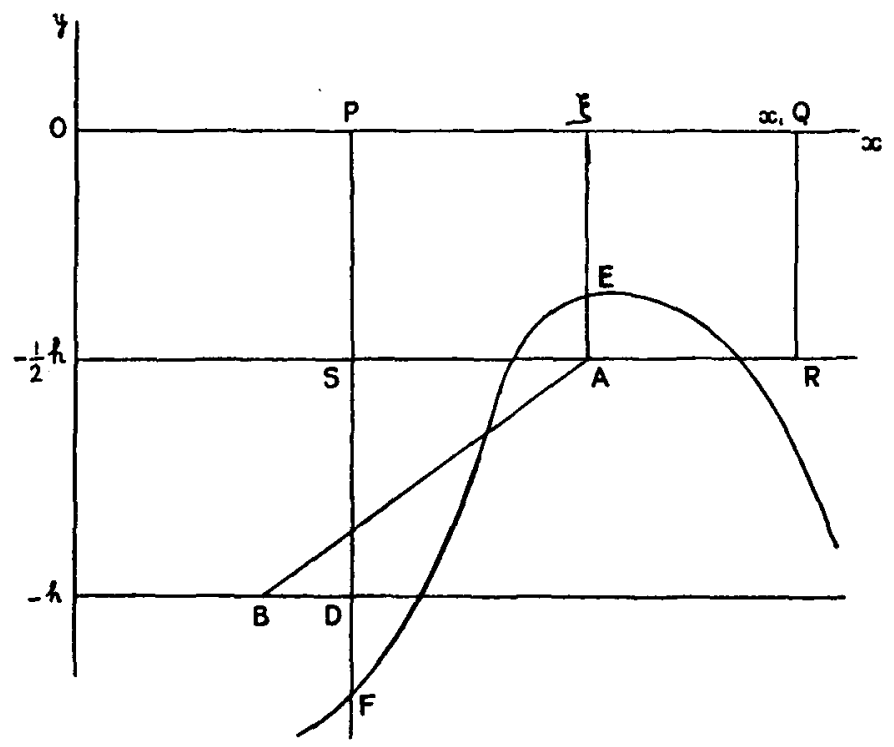

Fig. 2.

$\left(1-\frac{h}{4 K}\right) x_{1}$ and $x_{1}$ at which the curve rises above the line $y=-\frac{h}{2}$. For if this is not so it is obvious from the diagram (Fig. 2) that

$$
\int_{\left(1-\frac{h}{4 \bar{X}}\right) x_{1}}^{x_{1}} g(t) d t<\text { area of rectangle } P Q R S=-\frac{h^{2}}{8 K} x_{1}
$$

and we are led to a contradiction, as above.

Let $x_{1}$ be a point to the left both of $x_{0}$ and of $x$ such that at $x_{1}\left(1-\frac{h}{4 K}\right)$ the curve is below the line $y=-h$. Then we bave seen that there is a point $\xi, x_{1}\left(1-\frac{h}{4 K}\right)<\xi<x_{1}$, at which the 
curve is above the line $y=-\frac{h}{2}$. From $A\left(\xi,-\frac{h}{2}\right)$ draw a line $A B$ of slope $\frac{2 K}{\xi}$ cutting $y=-h$ in $B$. The abscissa of $B$ is

$$
\xi-\frac{h}{2} \cdot \frac{\xi}{2 K}=\xi\left(1-\frac{h}{4 K}\right)<x_{1}\left(1-\frac{h}{4 K}\right)
$$

so that $B$ lies to the left of $D$.

Thus, as is clear from the diagram, the curve must cut $A B$ in order to get from $E$ to $F$. This, as we have seen, contradicts $(\alpha)$. Thus hypothesis $(b)$ is untenable and the theorem is proved.

The argument is similar if we assume $(\beta)$ in place of $(\alpha)$.

Added 8th August 1925. 3. As an example consider the power series $\sum_{0}^{\infty} a_{n} x^{n} \equiv f(x)$, the coefficients $a_{n}$ being real, supposed convergent for $|x|<1$. As in Theorem 1 , let $f(x)$ possess an improper integral in $(0,1)$. Then we deduce.

Theorrm 2

$$
f(x) \rightarrow A \quad \text { as } x \rightarrow 1-0
$$

if

$$
\begin{aligned}
& \text { (i) } \frac{1}{1-x} \int_{x}^{1} f(t) d t \rightarrow A \\
& \text { (ii) } n a_{n}<K
\end{aligned}
$$

For, by (ii)

$$
\begin{aligned}
f^{\prime}(x) & =\sum_{1}^{\infty} n a_{n} x^{n-1}=(1-x)^{k} \sum_{1}^{\infty} S_{n}^{k} x^{n-1} \\
& <(1-x)^{k} \sum_{1}^{\infty} K A_{n}^{k} x^{n-1}=K(1-x)^{k}(1-x)^{-k-1} \\
& =\frac{K}{1-x}
\end{aligned}
$$

